\title{
INFINITE-DIMENSIONAL MANIFOLDS ARE OPEN SUBSETS OF HILBERT SPACE
}

\author{
BY DAVID W. HENDERSON ${ }^{1}$
}

Communicated by Richard Anderson, January 20, 1969

In this paper we prove, using Hilbert space microbundles, the

Theorem. If $M$ is a separable metric manifold modeled on the separable infinite-dimensional Hilbert space, $H$, then $M$ can be embedded as an open subset of $H$.

Each infinite-dimensional separable Fréchet space (and therefore each infinite-dimensional separable Banach space) is homeomorphic to $H$. (See [1].) We shall use " $F$-manifold" to denote "metric manifold modeled on a separable infinite-dimensional Fréchet space." Thus we have

Corollary 1. Each separable F-manifold can be embedded as an open subset of $H$.

Recent results of Eells and Elworthy [6] and Kuiper and Burghelea [9] and Moulis combine to show (see [6]) that two separable $C^{\infty}$ Hilbert manifolds are $C^{\infty}$-diffeomorphic if and only if they have the same homotopy type. Since open subsets of $H$ have an induced $C^{\infty}$ structure, we have

Corollary 2. Each F-manifold has a unique $C^{\infty}$-Hilbert manifold structure.

COROLlaRY 3. Two F-manifolds are homeomorphic if and only if they have the same homotopy type.

Results about open subsets of $H$ in [7] apply to give us

Corollary 4. For each F-manifold $M$ there is a countable locallyfinite simplicial complex $K$, such that $M$ is homeomorphic to $|K| \times H$.

Corollary 5. Each F-manifold is homeomorphic to an open set $U \subset H$, such that $H-U$ is homeomorphic to $H$ and $\operatorname{bd}(U)$ is homeomorphic to $U$ and to $\mathrm{cl}(U)$.

The author wishes to thank Marshall Cohen for suggesting the use of microbundles.

1. Hilbert microbundles. Milnor, in [11], defined the concept of (Euclidean) microbundles. We shall use strongly the ideas and defini-

1 The author is an Alfred P. Sloan Fellow. 
tions of [11]. In particular, we will use the terms microbundle, trivial microbundle, tangent microbundle, induced microbundle, and microbundle isomorphism exactly as on pp. 54-58 of [11] except that " $R^{n}$ " is replaced by " $H$ ". Presumably a whole theory of Hilbert microbundles can be developed, but in this paper we shall content ourselves with only proving what is necessary for the proof of the Theorem. Throughout this paper we will use " $\times 0$," " $p_{1}$ " and " $p_{2}$," to denote maps defined by setting $\times 0(b)=(b, 0), p_{1}(a, b)=a$, and $p_{2}(a, b)=b$. Detailed proofs will appear elsewhere.

Proposition 1. A microbundle $(B \stackrel{\rightarrow}{\rightarrow} \stackrel{\rightarrow}{\rightarrow} B)$ with paracompact base $B$, is trivial if and only if there is, for each open neighborhood $U$ of $i(B)$, an open neighborhood $V \subset U$ and a homeomorphism $h: V \rightarrow B \times H$ such that the following commutes:

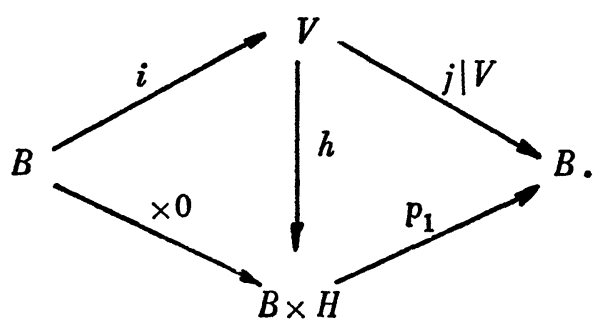

The proof is essentially the same as Lemma (2.3) of [11].

Proposition 2. Let $N$ be an open subset of $H$. Then the tangent microbundle $\mathrm{t}_{N}$ is trivial.

Proposition 3. Let $\mathfrak{x}(Y \dot{\rightarrow} \rightarrow E \stackrel{\rightarrow}{\rightarrow} Y)$ be a microbundle with $Y$ the union of two closed sets $A$ and $B$ such that $\mathfrak{x} \mid A$ and $\mathfrak{x} \mid B$ are trivial. If there is a retraction $r: B \rightarrow A \cap B$, then $\mathfrak{x}$ is trivial.

Proposition 4. Any microbundle $\mathfrak{b}(H \stackrel{i}{\rightarrow} E \stackrel{\dot{m}}{\rightarrow} H)$ with base space $H$ is trivial.

Proposition 5. Let $\mathfrak{b}(N \stackrel{i}{\rightarrow} E \stackrel{i}{\rightarrow} N)$ be a microbundle with base $N$, an open subset of $H$. Then there is an open neighborhood $W$ of $i(N)$ and a homeomorphism $k: W \rightarrow N \times H$ such that

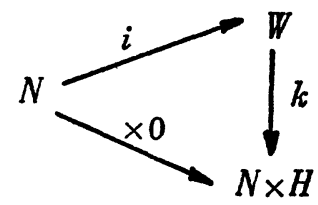

commutes. 
The proof of Proposition 5 hinges on the fact that, by a previous paper [7], $N$ is homeomorphic to $|K| \times H$, where $K$ is a countable locally-finite simplicial complex. The proof also uses infinite-dimensional results from [2], [3], and [8], and Propositions 3 and 4.

2. Proof of the theorem. Let $M$ be a separable manifold modeled on $H$ and let $i: M \rightarrow H$ be an embedding such that $i(M)$ is closed. A modification of $[\mathbf{5}$, p. 79] shows that such embeddings exist. Identify $M$ with $i(M)$. Since $M$ is an ANR $[5$, p. 99], there is a neighborhood $N$ of $i(M)=M$ for which there is a retraction $r: N \rightarrow M$. Let $h: U \rightarrow N \times H$ be the homeomorphism and open set given by the Proposition 2, which shows that $\mathrm{t}_{N}$ is trivial. Then $h^{-1}$ takes $M \times H$ homeomorphically onto $U \cap(M \times N)$ which is a neighborhood of $\Delta(M)$ in $M \times N$. The induced microbundle $r^{*}\left(\mathrm{t}_{M}\right)$ is

$$
N \stackrel{1, r}{\longrightarrow} N \times M \stackrel{p_{1}}{\longrightarrow} N .
$$

Since

$$
\begin{aligned}
& M \stackrel{\Delta}{\longrightarrow} M \times N \\
& \downarrow i \quad \stackrel{ }{\downarrow} \times\left(p_{2}, p_{1}\right) \\
& N \stackrel{(1, r)}{\longrightarrow} N \times M
\end{aligned}
$$

commutes, where $\left(p_{2}, p_{1}\right)(m, n)=(n, m)$, then, for $\phi \equiv\left(p_{2}, p_{1}\right) \circ h^{-1}$, $\phi(M \times H)$ is a neighborhood of $((1, r) \circ i)(M)=\left(\left(p_{2}, p_{1}\right) \circ \Delta\right)(M)$ in $N \times M$. By Proposition 5, there is an open set $W \subset N \times M$ such that $(1, r)(N) \subset W$ and $k:(W,(1, r)(N)) \rightarrow(N \times H, N \times\{0\})$ is a homeomorphism. Then $\phi^{-1}(W)$ is a neighborhood of $M \times\{0\}$ in $M \times H$ and thus by standard arguments (same as proof of Proposition 1) there is an embedding $\psi: M \times H \rightarrow W$ such that $\psi(M \times H)$ is open in $W$. But then $(k \circ \psi)(M \times H)$ is open in $N \times H$ which is open in $H \times H$ which is homeomorphic to $H$. Thus $M \times H$ can be embedded as an open subset of $H$. By a recent result of Anderson and Schori [4], $M$ is homeomorphic to $M \times H$, thus the theorem is proved.

\section{REFERENCES}

1. R. D. Anderson, Hilbert space is homeomorphic to the countable product of real lines, Bull. Amer. Math. Soc. 72 (1966), 515-519.

2. - On topological infinite deficiency, Michigan Math. J. 14 (1967), 365-383.

3. R. D. Anderson and R. H. Bing, A complete elementary proof that Hilbert space is homeomorphic to the countable infinite product of lines, Bull. Amer. Math. Soc. 74 (1968), 771-792.

4. R. D. Anderson and R. Schori, Factors of infinite-dimensional manifolds, Trans. Amer. Math. Soc. (to appear). 
5. Karol Borsuk, Theory of retracts, PWN, Warsaw, 1967.

6. J. Eells, Jr. and K. D. Elworthy, On the differential topology of Hilbertian manifolds, Proc. Summer Institute on Global Analysis, Berkeley, Calif., 1968.

7. D. W. Henderson, Open subsets of Hilbert space, Compositio Math. (to appear).

8. V. L. Klee, Convex bodies and periodic homeomorphism in Hilbert space, Trans. Amer. Math. Soc. 74 (1953), 10-43.

9. N. H. Kuiper and D. Burghelea, Hilbert manifolds (to appear).

10. E. Michael, Local properties of topological spaces, Duke Math. J. 21 (1954), 163-172.

11. J. Milnor, Microbundles. Part I, Topology 3 (1964), 53-80.

Cornell University, Ithaca, New York 14850 